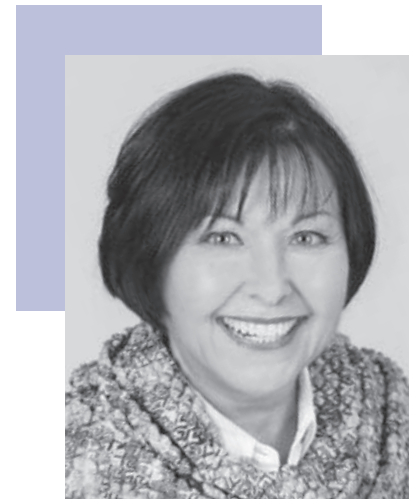

\title{
Editorial
}

\section{Übung macht den Meister - nur im Sport nicht}

Das deutsche Handwerk mit dem berühmten „goldenen Boden“ ist ein weltweit gültiges Gütezeichen. Im „Megatrend Gesundheit“ breitet sich dieser goldene Boden derzeit im Bereich des Gesundheitswesens aus. „Sport und Bewegung“ werden mehr denn je nicht nur Versorgungselemente im Gesundheitswesen, sondern auch Wirtschaftsfaktoren. Gepusht wird diese Entwicklung durch die Ratlosigkeit in der Politik angesichts des demografischen Wandels und der damit verbundenen Kostenentwicklung. Das Thema „Späterer Renteneintritt und Gesunderhaltung“ ist mittlerweile auch im Finanzministerium angekommen.

Der große gesellschaftliche und gesundheitsökonomische Bedarf an evidenzbasierter Bewegung und Bewegungsförderung wird immer deutlicher - nicht von ungefähr entwickelt das Bundesministerium für Gesundheit gerade „Nationale Empfehlungen für Bewegung und Bewegungsförderung“. Daran anknüpfend wird die große Aufgabe darin bestehen, die nahezu unüberschaubare Vielfalt an Tätigkeitsfeldern „Sport und Bewegung" zu analysieren, zu sichten und strukturiert zu vernetzen. Daran knüpft sich die Analyse und strukturierte Vernetzung der Akteure und Akteurinnen der Bewegung und Bewegungsförderung an - mit deren Qualifikationsentsprechungen.

Diese Sichtung wurde durch das BOLOGNA-Verfahren immens erschwert. Der genannte große Bedarf an Bewegung und Bewegungsförderung macht eine Professionalisierung dringend notwendig: Aus dem Tätigkeitsfeld Sport und Bewegung muss ein Beruf entstehen (vgl. Seite 128). Qualifikationsziele und Kompetenzen können aber nur Bildungsstätten vermitteln, die selbst qualifiziert und kompetent sind. Und hier liegt der Hase im Pfeffer: Mit dem Megatrend Gesundheit, Sport und Bewegung drängen kommerzialisierte Anbieter und Ausbilder in den Markt, die weder qualifiziert noch kompetent sind. Professionalisierung braucht klare Kompetenzhierarchien - und die fehlen im Tätigkeitsfeld „Bewegung und Sport“ eindeutig. Das Gefälle an Qualität und Kompetenzen ist im Sport ausgeprägt.

Die Nationale Präventionskonferenz wird sich bei Umsetzung der Bundesrahmenempfehlungen Prävention entscheiden müssen, ob sie politische Wirtschaftsinteressen Qualitätskriterien vorziehen wird. Professionalisierte Bewegungsfachkräfte brauchen eine Identität, die ihnen die Modulvielfalt der Hochschulen und Fachhochschulen derzeit nicht mehr geben kann. In der Gesundheitsversorgung (hier: Rehabilitation) werden Bewegungsangebote häufig durch andere Professionen „fremd besetzt“ - beispielsweise durch Mediziner oder Psychologen. Die Zeichen der Zeit hat auch der Fakultätentag Sportwissenschaft erkannt (vgl. Seite 158). Dieser gründete sich mit der Aufgabe, ein neues Memorandum zur Sportwissenschaft zu erstellen - und daraus ein Core Curriculum für die Sportwissenschaft zu definieren. Im Zusammenhang mit der Adaption einer EQR-Vorgabe wird dies eine mögliche Lösung des Kompetenz-Dilemmas bieten können.

Herzlichst Ihre

Angelika Baldus 